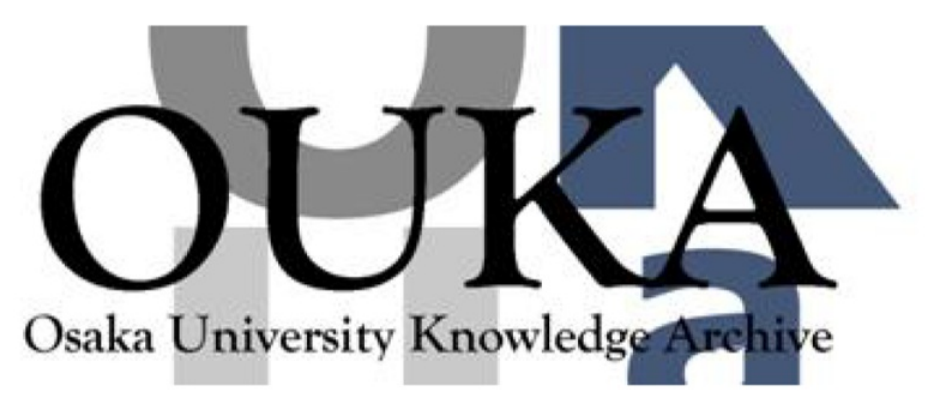

\begin{tabular}{|c|l|}
\hline Title & $\begin{array}{l}\text { Acoustic study of kinetics of vacancy diffusion } \\
\text { toward dislocations in aluminum }\end{array}$ \\
\hline Author(s) & $\begin{array}{l}\text { Ogi, Hirotsugu; Tsujimoto, Akihiro; Nishimura, } \\
\text { Shigeyuki et al. }\end{array}$ \\
\hline Citation & Acta Materialia. 53(2) p.513-p.517 \\
\hline Issue Date & $2004-11-17$ \\
\hline oaire:version & AM \\
\hline URL & $\begin{array}{l}\text { https://hdl. handle. net/11094/84189 } \\
\text { O2004 Acta Materialia Inc. Published by } \\
\text { Elsevier Ltd. This manuscript version is made } \\
\text { available under the Creative Commons } \\
\text { Attribution-NonCommercial-NoDerivatives 4.0 } \\
\text { International License. }\end{array}$ \\
\hline rights \\
\hline Note & \begin{tabular}{l} 
\\
\hline
\end{tabular} \\
\hline
\end{tabular}

Osaka University Knowledge Archive : OUKA

https://ir. Library. osaka-u. ac. jp/

Osaka University 


\title{
Acoustic Study of Kinetics of Vacancy Diffusion toward Dislocations in Aluminum
}

\author{
Hirotsugu Ogi, Akihiro Tsujimoto, Shigeyuki Nishimura, and Masahiko Hirao \\ Graduate School of Engineering Science, Osaka University \\ Machikaneyama 1-3, Toyonaka, Osaka 560-8531, Japan
}

\begin{abstract}
Using ultrasonic shear waves, we study the kinetics of migration of vacancy to dislocations in aluminum. Ultrasonic attenuation is sensitive to the effective dislocation length, which is shortened by the segregation of point defects. Two principal measurements were done: (i) measurement of temperature dependence of decay behavior of attenuation after elastic deformation, and (ii) continuous measurement of attenuation of a specimen quenched from $573 \mathrm{~K}$ to cold-water and then to liquid-nitrogen temperatures. The specimens were polycrystalline and monocrystal aluminum with 99.995\% purity. The former measurement provided us with the activation energy $0.28 \mathrm{eV}$ for migration of dominant point defects through the Granato-Hikata-Lücke theory. The latter measurement informed us of the kinetics of vacancy: the attenuation coefficient rapidly decreased twice at $125 \mathrm{~K}$ and $250 \mathrm{~K}$, corresponding to activation energies 0.28 and $0.61 \mathrm{eV}$ for migration, respectively. Thus, the same mechanism occurs for the attenuation change after elastic deformation and at 125 $\mathrm{K}$ after quenching. We attributed the $0.28 \mathrm{-eV}$ migration as fast diffusion of vacancy along dislocations. The 0.61-eV migration is consistent with the bulk diffusion of vacancy in aluminum. Throughout this study, the electromagnetic acoustic resonance method was used for making noncontacting and highly accurate measurement of attenuation.
\end{abstract}

Key words: acoustic methods, aluminum, diffusion, electromagnetic acoustic resonance, vacancies

62.40.+i, 61.72.Ji, 66.30.Lw, 81.70.Cv

Preprint submitted to Elsevier Science Received xx September; accepted xxxxxxx 


\section{INTRODUCTION}

Diffusion of vacancies occurs fast in aluminum because of the small activation energy for migration. It becomes faster near dislocations because a distorted lattice lowers the energy for migration(1). Therefore, it has been difficult to directly observe the kinetics of vacancy migration toward dislocations in aluminum. Electric-resistance methods have been conventionally used to study vacancy kinetics in aluminum $(2 ; 3 ; 4 ; 5)$, but these methods fail to detect vacancy diffusion for dislocations because of their unchanged number. In this study, we study the vacancy diffusion with two different acoustic methods to determine the activation energy of vacancy diffusion toward dislocations in aluminum for the first time. One is the measurement of isothermal decay of ultrasonic attenuation. The other is the measurement of ultrasonic attenuation after quenching.

Ultrasonic attenuation (or internal friction) has been used to study pointdefect diffusion near dislocations $(6 ; 7 ; 8 ; 9 ; 10 ; 11)$. It is proportional to a product of the dislocation density and the fourth power of the dislocation segment length (12). Point defects migrate and interact with dislocations to shorten their effective segment length, resulting in decrease of attenuation. Granato, Hikata, and Lücke (7) presented a theory (GHL theory) for explaining the decay behavior of ultrasonic attenuation due to the point-defect diffusion. Several researchers applied the GHL theory to study the kinetics of point-defect diffusion. However, because ultrasonic attenuation is highly affected by mechanical contacts needed for holding and gripping specimens, and for generating ultrasonic waves, the previous contact measurements were insufficient to analyze quantitatively the measurements in order to yield physical parameters related to the kinetics of point-defect diffusion. Energy losses due to the contacts are usually much larger than the change of attenuation caused by point-defect diffusion.

Recently, a noncontacting ultrasonic measurement, called electromagnetic acoustic resonance (EMAR), has been developed for accurately measuring ultrasonic attenuation $(13 ; 14 ; 15 ; 16)$. It uses an electromagnetic acoustic coupling (Lorentz force or magnetostriction force) to avoid the energy losses associated with the mechanical contacts, providing absolute values of attenuation. With this technique, we showed that vacancies are dominant defects for pinning dislocations at room temperature (16). Here, using the EMAR method, we intend to determine the activation energy of vacancy diffusion in aluminum from two kinds of attenuation measurements for $4 \mathrm{~N} 5$ aluminum specimens. (i) We isothermally measure the attenuation decay after an elastic deformation. The temperature dependence of the decay parameter yields the activation energy of vacancy diffusion near dislocations. (ii) We continuously measure ultrasonic attenuation during the temperature-increase process after quenching the speci- 
men. Attenuation rapidly drops at two temperature regions, reflecting vacancy diffusion, from which we estimate corresponding activation energies. The activation energy determined by the former measurement shows good agreement with that estimated in the latter measurement.

\section{MEASUREMENTS}

\subsection{Decay of Attenuation after Elastic Deformation}

Figure 1 shows measurement setup for continuously measuring the shear-wave attenuation at $4.7 \mathrm{MHz}$. The plate-shape specimen was polycrystalline aluminum with 99.995 mass\% purity, measuring $50 \mathrm{~mm}$ by $6 \mathrm{~mm}$ by $1 \mathrm{~mm}$. The material was annealed at $473 \mathrm{~K}$ for $1 \mathrm{~h}$ before the measurement. The macroscopic yield strength was $15 \mathrm{MPa}$ at room temperature, which was determined from the stress where the macroscopic stress-strain curve departs from linearity. A tensile load was applied along the longitudinal direction of the specimen through fixtures and bearings to avoid the bending stress. A solenoid coil was set near the specimen with a 0.5 - $\mathrm{mm}$ liftoff from the specimen surface. The specimen and coil were located in a furnace to control temperature, and a pair of permanent magnets was located outside the furnace to apply a static magnetic field needed for the Lorentz-force coupling (14). When we apply a sinusoidal current to the coil, eddy currents are generated, which interact with the static magnetic field to generate tangential Lorentz forces. The Lorentz forces generate the shear wave polarized parallel to the surface and normal to the loading direction, propagating along the thickness direction. The same coil detects the shear wave via the reversed Lorentz-force mechanism. Details to measure the resonance frequency and attenuation coefficient are given in Refs 13 and 14 . We continuously measured the attenuation coefficient during the stress sequence below:

(i) We applied a constant tensile stress of $4.9 \mathrm{MPa}$ for $30 \mathrm{~min}$ after the temperature became stable at a target value.

(ii) We increased the stress up to $9.8 \mathrm{MPa}$ with a rate of $0.01 \mathrm{MPa} / \mathrm{s}$.

(iii) We kept the 9.8-MPa stress unchanged for $30 \mathrm{~min}$ and reduced it to 4.9 $\mathrm{MPa}$ with the same rate.

(iv) We kept the 4.9-MPa stress unchanged for 30 min and then changed the specimen temperature.

The attenuation-coefficient measurements were done at various temperatures between 293 and $383 \mathrm{~K}$. 


\subsection{Attenuation Measurement after Quenching}

Two kinds of specimens were used. One was a rectangular parallelepiped polycrystalline aluminum with 99.995 mass\% purity. The other was an oriented rectangular parallelepiped monocrystal aluminum with 99.995 mass\% purity. They measured 1.0x6.0x5.0 $\mathrm{mm}^{3}$.

A two-step quenching method was used: The specimen was heated up to 573 $\mathrm{K}$ in a furnace and kept there for $1 \mathrm{~h}$. It was dropped into a water bath kept at $277 \mathrm{~K}$ through a window on the furnace bottom, which was manually opened. This procedure took $0.5 \mathrm{~s}$. After removing water on the specimen surfaces, we threw it into a liquid-nitrogen bath where a solenoid coil and surrounding permanent magnets were located. A brass guide led the specimen into the solenoid coil. This procedure took $30 \mathrm{~s}$. Thus, free vibrations were caused by the electromagnetic acoustic coupling when we applied a sinusoidal current to the coil (17). We increased the temperature in the nitrogen bath with a rate of $30 \mathrm{~K} / \mathrm{h}$ and measured the attenuation coefficient of a free-vibration mode at $1.55 \mathrm{MHz}$ after the nitrogen evaporation.

For comparison, we performed non-quenching measurements: The specimen was heated up to $573 \mathrm{~K}$, held at this temperature for $1 \mathrm{~h}$, and then cooled in the furnace. It was thrown into the water bath and then into the liquidnitrogen bath after removing water on the surfaces by the same procedure as the quenching measurements. We then measured the attenuation as a function of increasing temperature.

\section{RESULTS}

Figure 2 shows a typical attenuation behavior during the stress sequence. The attenuation coefficient rapidly increased just after each constant-stress term and it decreased with time to the value before the stress was fixed during the next constant-stress term. Figure 3 plots the change of the attenuation coefficient during the constant-stress term at $4.9 \mathrm{MPa}$ at various temperatures. The decay rate increased as temperature increased until $333 \mathrm{~K}$ but it decreased beyond the temperature. This trend was commonly observed when we kept the stress at $9.8 \mathrm{MPa}$.

Figure 4 shows examples of the quenching and non-quenching measurements. From these measurements, we made the following common observations:

(i) The attenuation coefficient rapidly dropped near 125 and $250 \mathrm{~K}$ in the quenched specimens. 
(ii) It remained nearly unchanged in the non-quenched specimens.

(iii) Attenuation of the quenched monocrystal was larger than that of the quenched polycrystal. Also, the attenuation decrease was more noticeable for the quenched monocrystal specimens.

We made electric resistance measurements for the quenched and non-quenched specimens. The resistance of the non-quenched specimen showed the monotonic increase with temperature, but that of the quenched specimen clearly showed a local minimum at $250 \mathrm{~K}$.

\section{DISCUSSIONS}

The attenuation behavior observed here can be understood by dislocation pinning by movable point defects. Dislocation annihilation and dislocation rearrangement might cause the attenuation change but they cannot explain the abrupt increase of attenuation at the beginning of the unloading process. Granato, Hikata, and Lücke (7) gave a theory (GHL theory) explaining the attenuation behavior after deformation, considering diffusion of point defects toward dislocations. Their result is given by

$$
\alpha=\frac{A_{1}}{\left(n_{1}+n_{2}\right)^{4}} \cdot \frac{1}{\left(1+\beta t^{2 / 3}\right)^{4}}+\alpha_{b}
$$

Here, $A_{1}$ is a constant relating to the damping constant and line tension of dislocations and it will be unchanged during constant-stress terms. $n_{1}$ and $n_{2}$ denote concentrations of moveable and immovable point defects, respectively, $t$ the time measured after the attenuation change starts, and $\beta$ the attenuationdecay parameter. We added a background attenuation $\alpha_{b}$ to compensate for the time-independent dislocation damping (that is, dislocation damping after complete recovery), grain-scattering loss, and diffraction loss (16). The first occurs because the number of point defects on a dislocation line saturates and the average-dislocation-segment length converges to a finite value at infinite time.

The decay parameter $\beta$ is given by

$$
\beta=B \frac{n_{1}}{n_{1}+n_{2}}\left(\frac{D}{T}\right)^{\frac{2}{3}}
$$

Here, $T$ denotes temperature and $B$ is a constant, depending on the lattice parameter. $D$ is the diffusion coefficient of the movable point defects and it 
takes the form

$$
D=D_{0} \exp \left(-\frac{E_{m}}{k T}\right)
$$

Here $D_{0}$ denotes the diffusion coefficient at the high-temperature limit, $E_{m}$ the activation energy for movement, and $k$ Boltzmann's constant. Thus, measurement of the attenuation behavior after elastic deformation at various temperatures provides us with kinetics of the point defects movability.

We determined the attenuation-decay parameters by fitting Eq. (1) to our measurements. Solid lines in Fig. 3 are fitted theoretical results, showing good agreement with the measurements. Figure 5 shows $\beta$ versus temperature when the stress was kept at $4.9 \mathrm{MPa}$. $\beta$ increased with temperature but decreased beyond $333 \mathrm{~K}$. We attribute the decrease of $\beta$ with temperature to dislocation movements in the presence of a biasing stress. Because the stress remained unchanged, dislocations continuously moved, dragging the point defects and keeping their effective segment length long. Bremnes et al. (11) suggested that such a dislocation movement occurred at temperatures higher than 335 $\mathrm{K}$; they measured the attenuation response by applying a cyclic stress. The attenuation-stress curve converges to a closed loop (signature) that characterizes the interaction mechanism. Beyond $335 \mathrm{~K}$, the closed loop shows a figure-of-eight signature which suggests dislocation movements dragging point defects, being consistent with our result. Because we are interested only in the point-defect kinetics, we used the $\beta$ values below $333 \mathrm{~K}$.

Equations (1)-(3) indicate that the activation energy can be determined by logarithmically plotting $T \beta^{3 / 2}$ versus $1 / k T$, which yields a line of slope $E_{m}$. Figure 6 shows such plots, resulting in $E_{m}=0.28 \pm 0.03 \mathrm{eV}$ for both constant stresses of 4.9 and $9.8 \mathrm{MPa}$. This value is considerably smaller than the activation energy of vacancy's bulk diffusion in aluminum $(0.61-0.65 \mathrm{eV}$ $(2 ; 4)$ ) and larger than that of diffusion of self-interstitials in aluminum ( 0.1 $\mathrm{eV}(15)$ ). As shown below, this activation energy is linked with the quenching measurements.

Quenched specimens show larger attenuation because of dislocations caused by the different quenching rates between surface and inner part of the specimen. The fact that non-quenched specimens show no remarkable attenuation change over the temperature range supports our view that the rapid decreases of attenuation near 125 and $250 \mathrm{~K}$ in the quenched specimens are caused by vacancy diffusion toward dislocations. The smaller changes of attenuation in the polycrystalline specimens than in the monocrystal specimens are explained by the smaller number of vacancies in the polycrystalline specimens, because grain boundaries can be their sinks and allow them to recover during the quenching procedure. The higher-recovery temperature $(250 \mathrm{~K})$ corresponds 
to the temperature at which the electric resistance dropped. Thus, this indicates recovery of vacancies. Garr and Sosin (4) observed recovery of electric resistance at this temperature in a $4 \mathrm{~N} 5$ aluminum to which irradiation was applied at $4 \mathrm{~K}$. Thus, at this temperature, bulk diffusion of vacancies occurs. We attribute the lower-recovery temperature $(125 \mathrm{~K})$ to the fast diffusion of vacancies near dislocations. Considering that the electric resistance did not show a drop near this temperature, vacancies diffusion near dislocations cannot be detected by the conventional electric-resistance method because their number is unchanged.

We estimate the temperature dependence of movability of vacancies assuming activation energies determined by the attenuation-decay measurement above and that for bulk diffusion $(=0.61 \mathrm{eV})$. The jump frequency $f$ of a point defect is given by

$$
f=Z \nu \exp \left(-\frac{E_{m}}{k T}\right)
$$

where $Z$ denotes the coordination number and it equals 12 for an f.c.c. material. $\nu$ is the atomic-vibration frequency and of the order of $10^{12} \mathrm{~s}^{-1}$ for aluminum (18). Taking $\nu=5 \times 10^{12} \mathrm{~s}^{-1}$, we calculated the temperature dependence of the jump frequency, which is shown by broken lines in Fig. 4. The rapid decreases of attenuation at $125 \mathrm{~K}$ and $250 \mathrm{~K}$ approximately corresponds to activation energies $0.28 \mathrm{eV}$ and $0.61 \mathrm{eV}$, respectively, strongly indicating that the attenuation decrease at $125 \mathrm{~K}$ is caused by vacancy diffusion toward dislocations. Thus, different acoustic measurements yielded identical result in kinetics of point defects because of high accuracy of the attenuation measurements.

\section{CONCLUSIONS}

We studied diffusion of vacancies in aluminum with noncontacting ultrasonic measurements. Attenuation decay after an elastic deformation depended on temperature and the temperature dependence of the decay parameter yielded an activation energy of $0.28 \pm 0.03 \mathrm{eV}$. The attenuation measurement for quenched specimens detected abrupt decreases of attenuation near $125 \mathrm{~K}$ and $250 \mathrm{~K}$, corresponding to activation energies 0.28 and $0.61 \mathrm{eV}$ for migration, respectively. The lower-temperature drop of attenuation cannot be detected by the electric-resistance measurement. Thus, the $0.28-\mathrm{eV}$ migration indicates fast diffusion of vacancies toward dislocations. 


\section{References}

[1] Friedel J. Dislocations. New York:Pergamon Press; 1964.

[2] Desorbo W, Turnbull D. Phys Rev 1959;115:560.

[3] Garr KR, Sosin A. Phys Rev 1967;162:669.

[4] Garr KR, Sosin A. Phys Rev 1967;162:681.

[5] Schilling W, Sonnenberg K. J Phys F: Metal Phys 1973;3:322.

[6] Hikata A, Truell R, Granato A, Chick B, Lücke K. J Appl Phys 1956;27:396.

[7] Granato A, Hikata A, Lücke K. Acta Met 1958;6:470.

[8] Phillips DC, Pratt PL. Phil Mag 1970;21:217.

[9] Anderson AR, Pollard HF. J Appl Phys 1979;50:5262.

[10] Gremaud G, Bujard M, Benoit W. J Appl Phys 1987;61:1795.

[11] Bremnes Ø, Progin O, Gremaud G, Benoit W, Phys Stat Sol (a) 1997;160:395.

[12] Granato A, Lücke K. J Appl Phys 1956;27:583.

[13] Hirao M, Ogi H. EMATs for Science and Industry: Noncontacting Ultrasonic Measurements. Boston:Kluwer Academic; 2003.

[14] Ogi H, Suzuki N, Hirao M. Metal Mater Trans A 1998;29:2987.

[15] Johnson W. Mat Sci Eng A 2001;A309-310:69.

[16] Ogi H, Tsujimoto A, Hirao M, Ledbetter H. Acta Mater. 1999;47:3745.

[17] Ogi H, Ledbetter H, Kim S, Hirao M. J Acoust Soc Am 1999;106:660.

[18] Walker CB. Phys Rev 1956;103:547. 


\section{Figure Caption}

Fig. 1 Measurement setup for shear-wave attenuation by electromagnetic acoustic resonance technique.

Fig. 2 Evolution of the shear-wave attenuation coefficient during the loadingunloading stress sequence for 99.995 mass $\%$ polycrystalline aluminum. Frequency was near $4.7 \mathrm{MHz}$.

Fig. 3 Changes of the attenuation coefficient when the polycrystalline-aluminum specimen was loaded with the constant stress of $4.9 \mathrm{MPa}$ at various temperatures. Solid lines are fitted functions of Eq. (1). $\alpha_{0}$ denotes the attenuation coefficient at the beginning of the constant-stress loading.

Fig. 4 Changes of the attenuation coefficient measured after quenching the specimens from $573 \mathrm{~K}$ to cold-water temperature and then to liquid-nitrogen temperature. The measurement for the non-quenched specimen is also shown. Broken curves are estimated jump frequency $(f)$ for activation energies 0.28 and $0.61 \mathrm{eV}$.

Fig. 5 Temperature dependence of the attenuation-decay parameter $\beta$ obtained from the results for 99.995 mass $\%$ polycrystalline aluminum.

Fig. 6 Logarithmic plot of $T \cdot \beta^{3 / 2}$ versus $1 / k T$ obtained from the results for 99.995 mass $\%$ polycrystalline aluminum. Slopes give the activation energy $E_{m}$. 


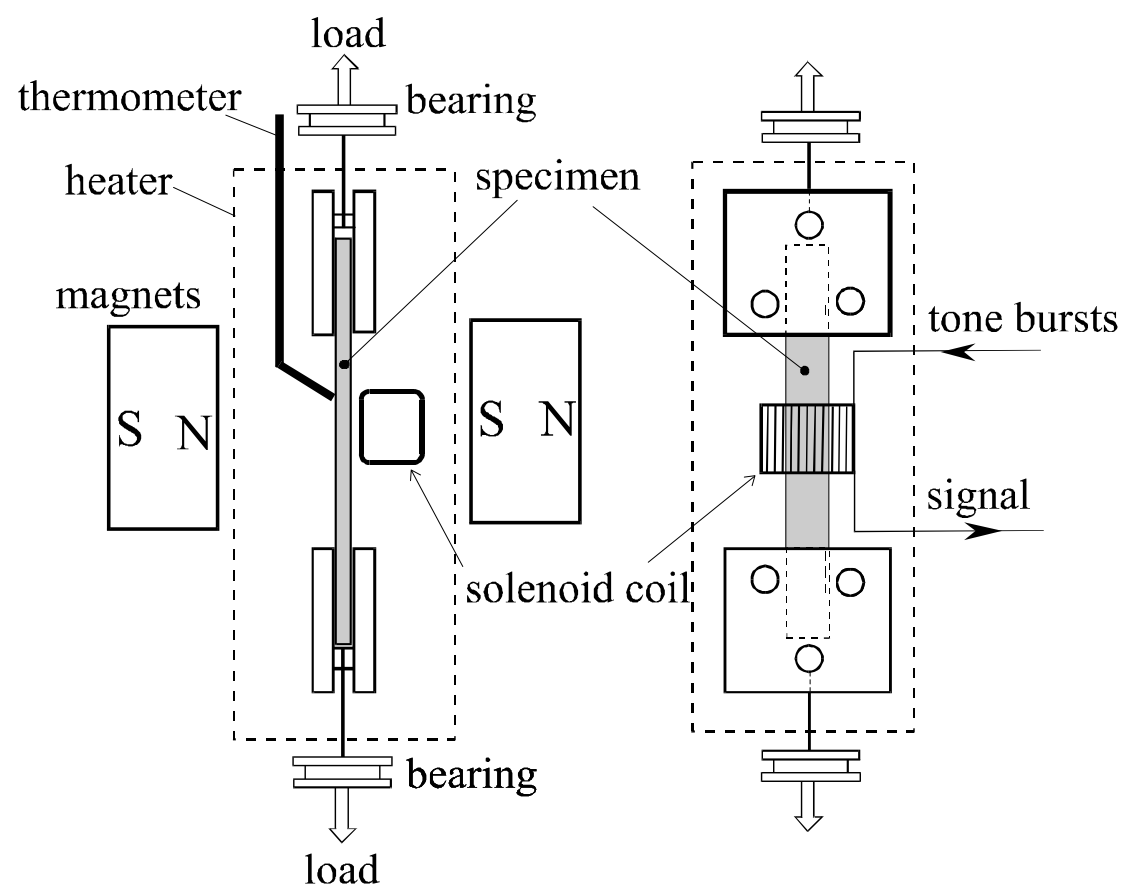

Fig. 1. Measurement setup for shear-wave attenuation by electromagnetic acoustic resonance technique. 


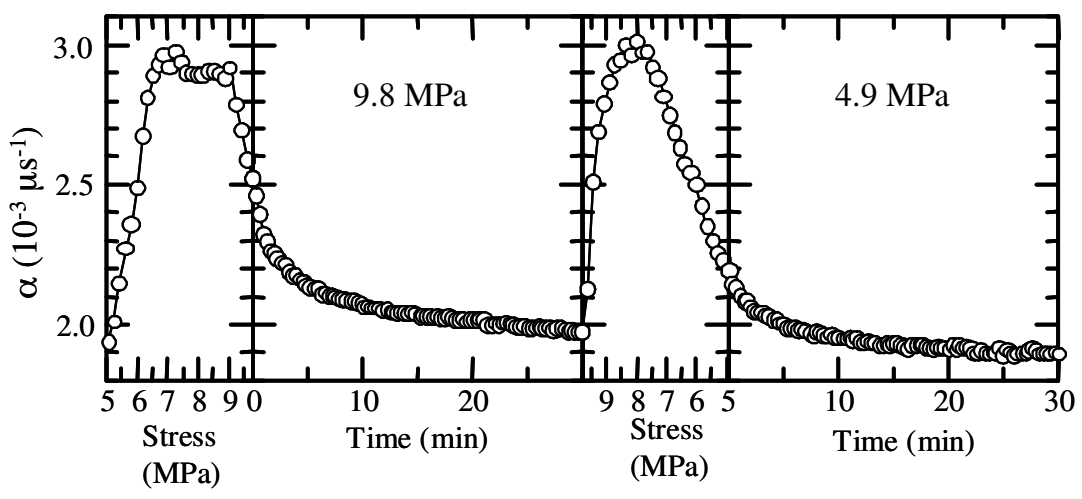

Fig. 2. Evolution of the shear-wave attenuation coefficient during the loading-unloading stress sequence for 99.995 mass \% polycrystalline aluminum. Frequency was near $4.7 \mathrm{MHz}$. 


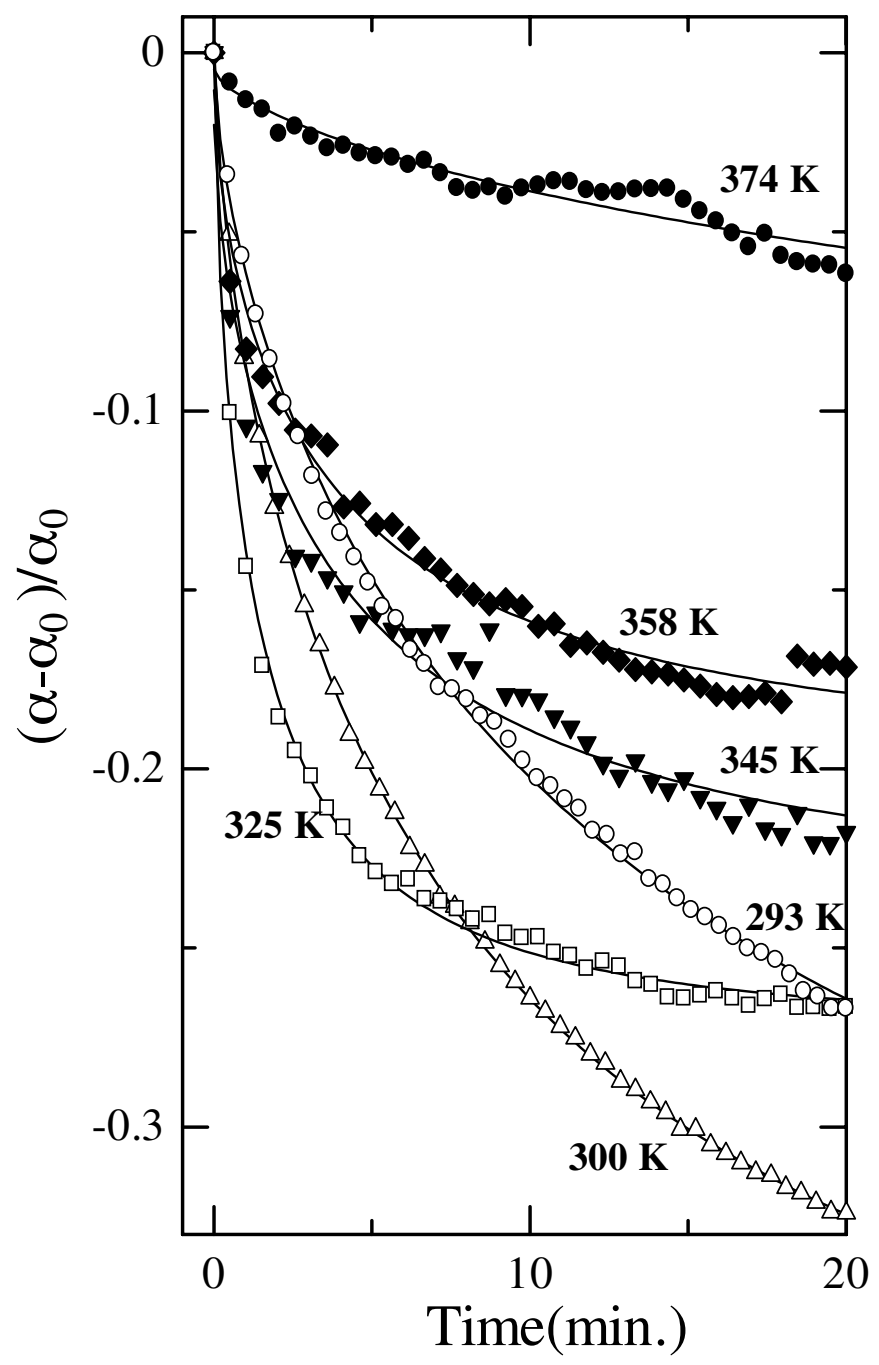

Fig. 3. Changes of the attenuation coefficient when the polycrystalline-aluminum specimen was loaded with the constant stress of $4.9 \mathrm{MPa}$ at various temperatures. Solid lines are fitted functions of Eq. (1). $\alpha_{0}$ denotes the attenuation coefficient at the beginning of the constant-stress loading. 


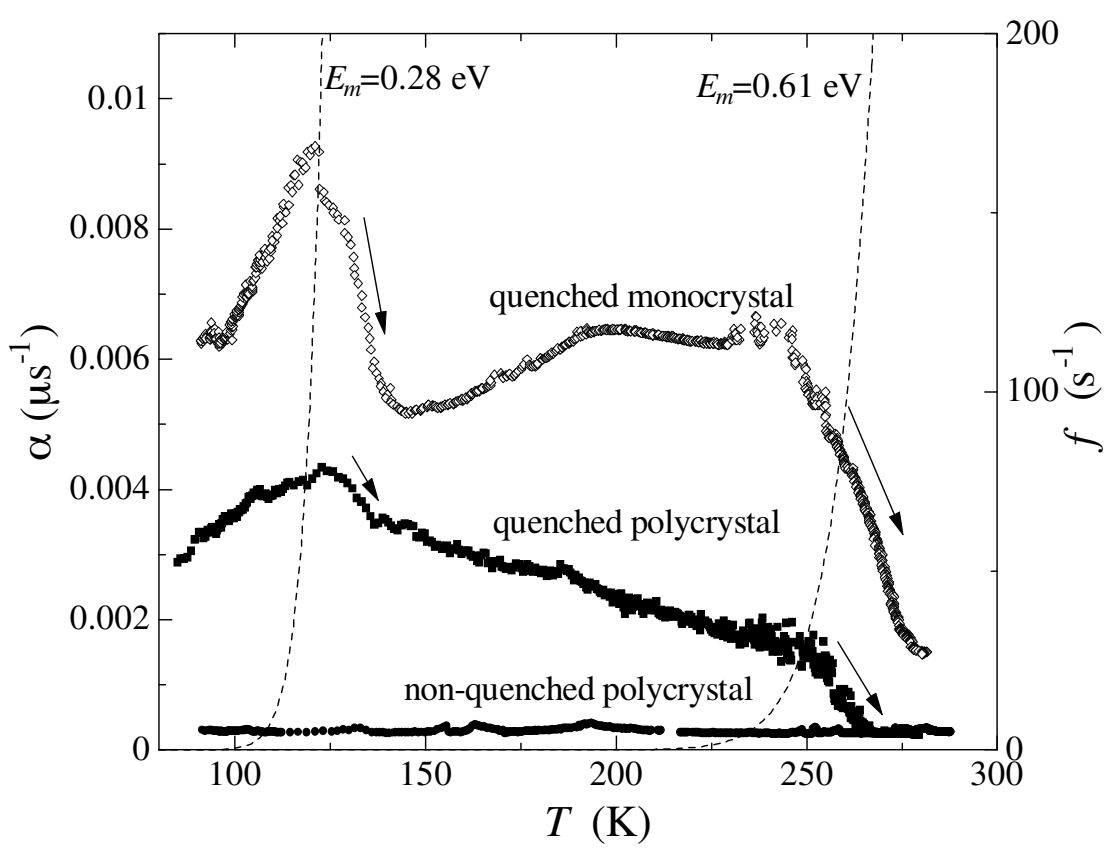

Fig. 4. Changes of the attenuation coefficient measured after quenching the specimens from $573 \mathrm{~K}$ to cold-water temperature and then to liquid-nitrogen temperature. The measurement for the non-quenched specimen is also shown. Broken curves are estimated jump frequency $(f)$ for activation energies 0.28 and $0.61 \mathrm{eV}$. 


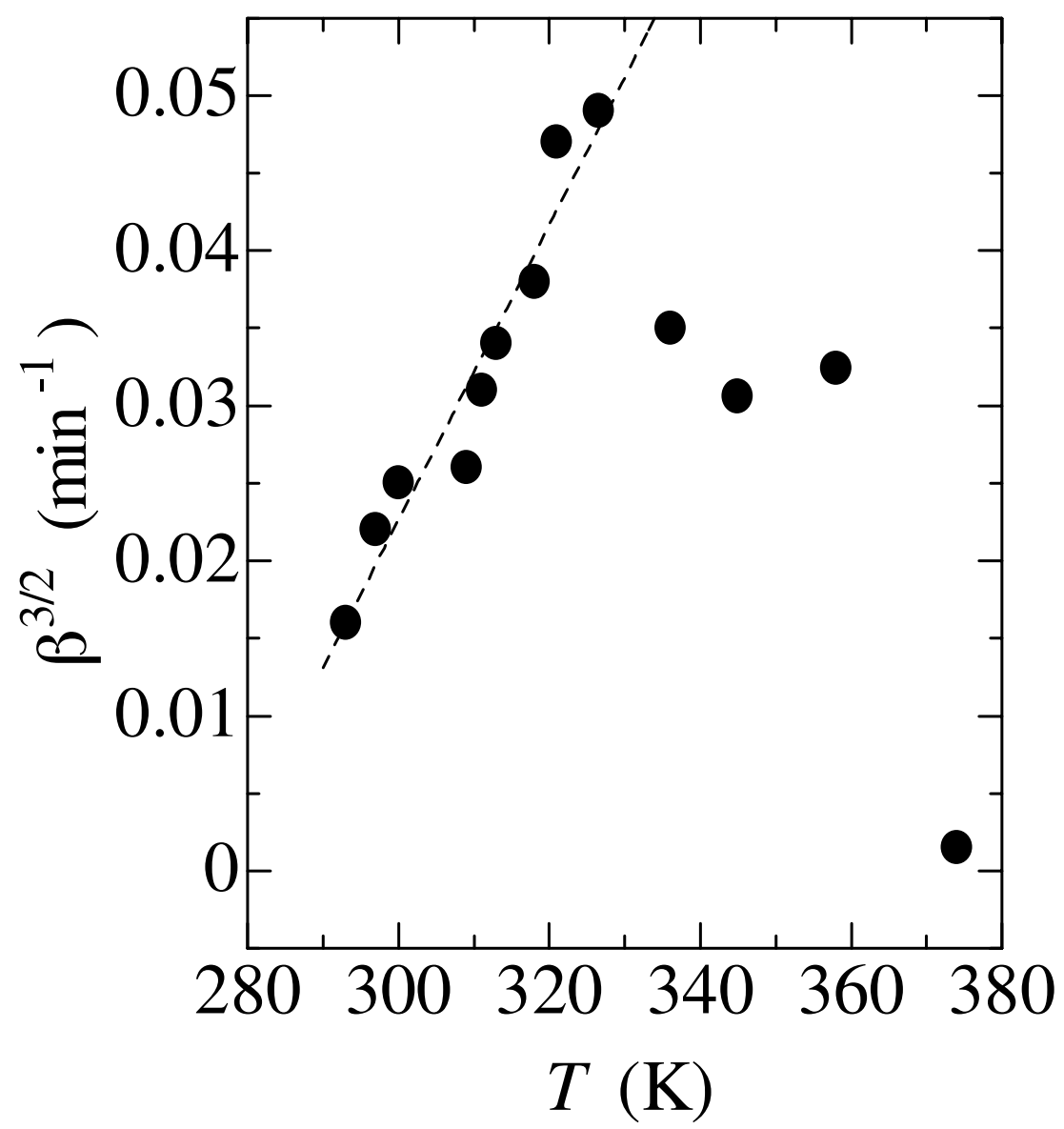

Fig. 5. Temperature dependence of the attenuation-decay parameter $\beta$ obtained from the results for 99.995 mass\% polycrystalline aluminum. 


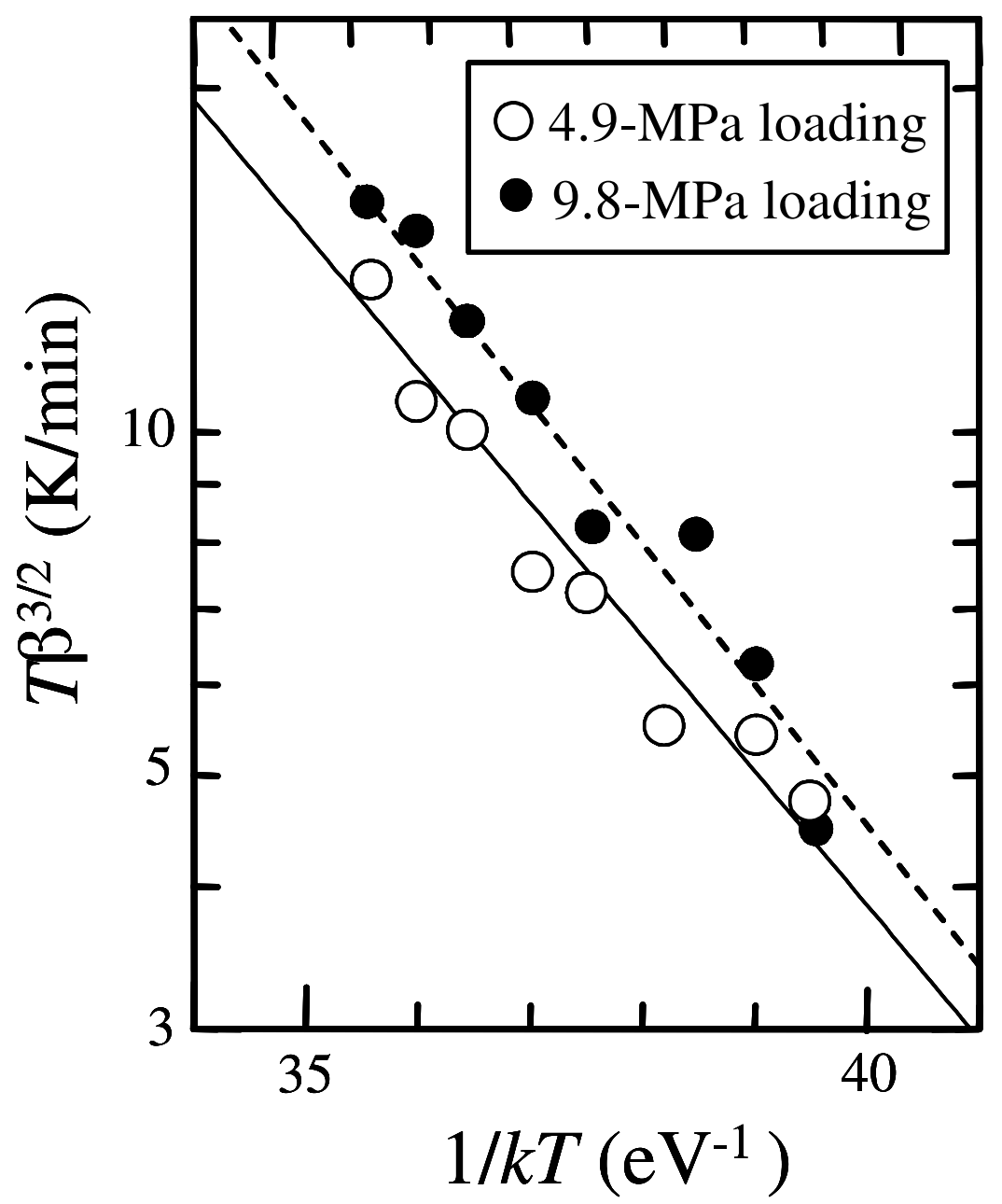

Fig. 6. Logarithmic plot of $T \cdot \beta^{3 / 2}$ versus $1 / k T$ obtained from the results for 99.995mass\% polycrystalline aluminum. Slopes give the activation energy $E_{m}$. 